Case Report

\title{
Adalimumab-associated Pulmonary Cryptococcosis
}

\author{
Takashi Iwata, MD, PhD, ${ }^{1,2}$ Teruaki Nagano, $\mathrm{MD},{ }^{3}$ Masuhiro Tomita, MD, PhD, ${ }^{4}$ \\ Yasuo Suehiro, MD, ${ }^{1,2}$ Shin-ichi Nakatsuka, MD, PhD ${ }^{3}$ Hayato Kimura, MD, PhD, ${ }^{3}$ \\ and Masayuki Sakaki, MD, $\mathrm{PhD}^{2}$
}

\begin{abstract}
This is the first report of adalimumab-associated pulmonary cryptococcosis. A 56-year-old female with rheumatoid arthritis without a history of pulmonary disease was simultaneously administered adalimumab $(40 \mathrm{mg} / 2 \mathrm{wks})$, methotrexate $(4 \mathrm{mg} / \mathrm{wk})$, and isoniazid $(200 \mathrm{mg} /$ day). Five months later, chest radiography revealed a small spiculated pulmonary nodule, and the laboratory test results, including levels of tumor markers and plasma $\beta$-D-glucan, were within normal ranges. Since the lesion continued to grow, even after discontinuing adalimumab, it was surgically resected. Grocott staining of the tissue sample revealed blackbrown fungi, identified as Cryptococcus neoformans in culture. The patient now remains well, without adalimumab therapy.
\end{abstract}

Key words: tumor necrosis factor, alpha, cryptococcosis, lung, adalimumab, surgery

\section{Introduction}

Monoclonal antibody therapy targeting tumor necrosis factor-alpha $(\mathrm{TNF}-\alpha)$ is a recent breakthrough in the treatment of rheumatoid arthritis (RA) and other autoimmune diseases. Adalimumab is the first, fully human, recombinant, IgG1 monoclonal antibody that specifically binds to human TNF- $\alpha$ and modulates its biological functions and suppresses proinflammatory reactions. Adalimumab has been approved for RA in the US market since December 2002, in Europe, since September 2003,

\footnotetext{
${ }^{1}$ Department of Thoracic Surgery, Osaka City University Graduate School of Medicine, Osaka, Osaka, Japan

${ }^{2}$ Departments of Thoracic and Cardiovascular Surgery, Kansai Rosai Hospital, Amagasaki, Hyogo, Japan

${ }^{3}$ Departments of Pathology, Kansai Rosai Hospital, Amagasaki, Hyogo, Japan

${ }^{4}$ Departments of Orthopedic Surgery, Kansai Rosai Hospital, Amagasaki, Hyogo, Japan
}

Received: March 8, 2010; Accepted: April 30, 2010

Corresponding author: Takashi Iwata, MD, PhD. Department of Thoracic and Cardiovascular Surgery, Kansai Rosai Hospital, 3-169 Inabaso, Amagasaki, Hyogo 650-8511, Japan

Email: taiwata@kanrou.net

(C)2011 The Editorial Committee of Annals of Thoracic and Cardiovascular Surgery. All rights reserved. and in Japan, since April 2008. Anti-TNF therapy sometimes shows dramatic improvement in the clinical symptoms of various autoimmune diseases such as RA; however, it is also known to increase the risk of various infectious diseases, especially tuberculosis, in immunocompromised patients. Fungal infections can also arise as complications during the therapy. Of the 28 cases of antiTNF therapy-associated Cryptococcus infection reported in the literature, ${ }^{1)} 17$ had received infliximab and $10 \mathrm{had}$ received etanercept; the only case that was associated with adalimumab demonstrated a cutaneous infection, resulting in the amputation of the infected finger. ${ }^{2)}$ Another case of adalimumab therapy-associated cryptococcal infection of a finger has also been reported. ${ }^{3)}$ However, deep cryptococcal infection associated with adalimumab therapy has never been reported to date. We report the first case of pulmonary cryptococcosis developing during combination therapy with adalimumab and methotrexate for RA and its successful treatment by surgical resection and discontinuation of anti-TNF therapy.

\section{Case Report}

The patient was a 56-year-old female with a 6-month history of RA. No evidence of a history of tuberculosis or 


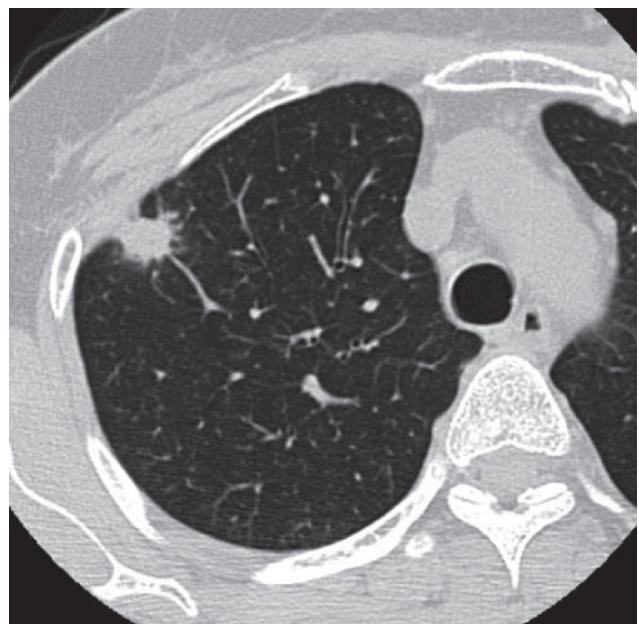

Fig. 1 Chest computed tomography showing a spiculated subpleural mass in the apical area of the right lung.

pulmonary shadow indicative of other conditions was detected on chest computed tomography (CT) performed before anti- TNF- $\alpha$ therapy. Isoniazid (200 mg/day) had been prophylactically started by the rheumatologist $1 \mathrm{wk}$ prior to the initiation of adalimumab (HUMIRA ${ }^{\circledR}$ ). Serum value of matrix metalloprotease 3 (MMP3), a marker of joint destruction that reflects the activity of RA, was $224 \mathrm{ng} / \mathrm{ml}$ (normal range; 17.3-59.7). Adalimumab was then started at the dose of $40 \mathrm{mg} / 2 \mathrm{wks}$. Five weeks later, $4 \mathrm{mg} / \mathrm{wk}$ of methotrexate was added. Steroids had never been administrated during the entire clinical course. Five months after the initiation of adalimumab, a small, nodular lesion was demonstrated in the chest radiograph. The rheumatologist suspected that the lung mass indicated tuberculosis; therefore, adalimumab treatment was discontinued immediately, while methotrexate and isoniazid were continued. She did not complain any symptoms of arthralgia at that time, with $50.4 \mathrm{ng} / \mathrm{ml}$ of serum MMP3 value. The patient was then transferred to our department for further evaluation and treatment of the lung shadow. Chest radiography and CT (Fig. 1) showed a spiculated subpleural mass about $1 \mathrm{~cm}$ in diameter, in the apical area of the right lung. Blood tests showed a white blood cell count of $4700 / \mu 1$, neutrophils $40.7 \%$, lymphocytes $47.3 \%$, monocytes $7.0 \%$, and eosinophils $4.3 \%$. The patient's serum was negative for tumor markers, such as carcinoembryonic antigen $1.9 \mathrm{ng} / \mathrm{ml}$ (cut off 5.0) and squamous cell carcinoma antigen $0.8 \mathrm{ng} / \mathrm{ml}$ (cut off 1.5). Plasma $\beta$-D-glucan was less than $5.0 \mathrm{pg} / \mathrm{ml}$ (cut off 20.0). The human immunodeficiency virus anti-

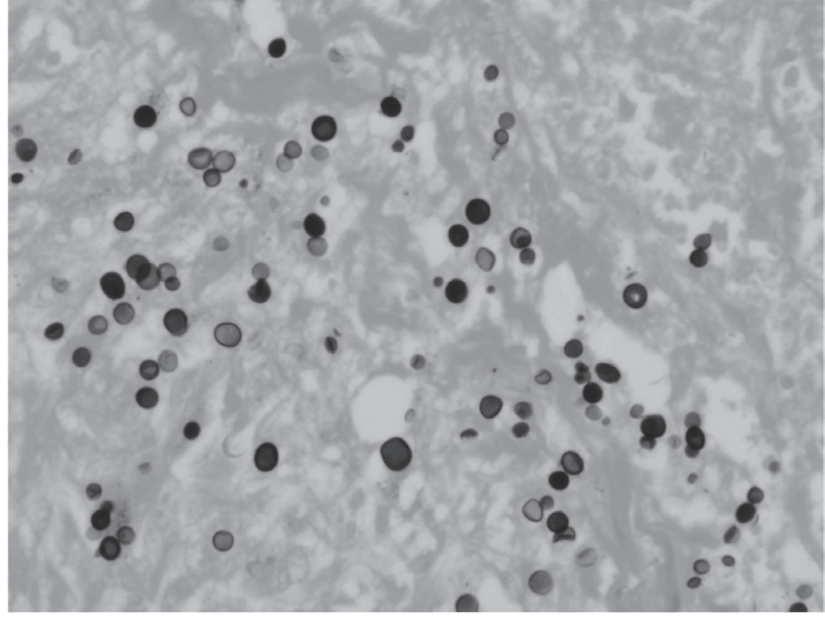

Fig. 2 Grocott staining of tissue specimen showing round, blackbrown, yeast-like fungi.

body test result was also negative. The patient was not diabetic, and other laboratory data were also within normal ranges. Two-[18F]-fluoro-2-deoxy-D-glucose positron emission tomography showed increased uptake in the pulmonary lesion with a maximum standard uptake value of 2.57. Systemic check-up did not show any metastatic lesions.

Because the lesion continued to grow in size gradually, even after $4 \mathrm{wk}$ of adalimumab discontinuation and isoniazid treatment, lung cancer, tuberculosis (especially the multidrug-resistant variety), diseases due to nontuberculous mycobacteria, or other infectious diseases were suspected. Therefore, the lung mass was surgically resected by wedge resection via video-assisted thoracoscopy. An analysis of intraoperative frozen sections revealed necrotic lesions, but no signs of malignancy. Postoperative pathological examination showed central necrosis and peripheral inflammation that mainly consisted of histiocytes. Multinucleated giant cells were observed focally. Grocott stain revealed black-brown fungi mainly in the macrophages (Fig. 2). Cryptococcus neoformans was identified in culture. The postoperative course was uneventful, and the patient recovered without the use of any further anti-fungal agents during the entire clinical course; isoniazid was also discontinued. Adalimumab therapy was suspended, and RA is currently treated only by methotrexate $6 \mathrm{mg}$ a day. Arthralgia was exacerbated two months after surgery, with mild elevation of serum MMP3 value to $127 \mathrm{ng} / \mathrm{ml}$. However, the symptoms were almost disappeared 6 months after surgery, with serum 
MMP3 value descent to $85.7 \mathrm{ng} / \mathrm{ml}$. Because a railway station on the patient's commuting route was found polluted by excretory substance of doves, adalimumab was still suspended and not being reviewed to start again.

\section{Discussion}

TNF- $\alpha$ antagonists are a part of a new and revolutionary treatment for severe and difficult-to-treat autoimmune diseases, including RA, juvenile idiopathic arthritis, psoriatic arthritis, plaque psoriasis, ankylosing spondylitis, and Crohn's disease. However, these agents can also imperil the patient's immunity to tuberculosis and fungal infections. ${ }^{1,4)}$ T-cell immunity plays a central role in protecting and clearing Cryptococcus infections from the lung. ${ }^{5,6)}$ Thus, TNF- $\alpha$ antagonists that suppress T-cell immunity increase the risk of cryptococcosis and other opportunistic infections. Recently, the US Food and Drug Administration (FDA) issued repeated warnings against the use of anti-TNF therapy because of the associated risk of serious opportunistic fungal infections.7) In its reports, the FDA had reviewed 240 patients with histoplasmosis who were treated with various TNF- $\alpha$ antagonists, such as etanercept, infliximab, and adalimumab. Most of the patients resided in the Ohio River and Mississippi River Valleys, where the fungus is commonly found. In contrast, the distribution of Cryptococcus neoformans is not regional, and it is often found in the excreta of doves; therefore, exposure to contaminated environments should be avoided.

Methotrexate was also concomitantly administrated with adalimumab in this case. Methotrexate, an antimetabolite of folic acid, also has an immune suppressing effect, inhibiting antibody production and lymphocyte proliferation and possibly increasing the chance of opportunistic infection. Methotrexate has pulmonary toxicity itself and increases the chance of bacterial pneumonia, pneumocystis carinii pneumonia, cytomegalovirus and herpes zoster virus infection. However, comorbidity of pulmonary cryptococcosis was merely reported as a complication of methotrexate monotherapy without concomitant use of corticosteroid, and we could find only two cases in the literature. ${ }^{89}$ Thus, we thought that adalimumab might play a more important role in the complication of pulmonary cryptococcosis than by methotrexate in this case.

We only have a 7-year-experience for the clinical use of adalimumab in the US, 6 years in Europe, and 1 year in Japan. Reports from Spain showed no cryptococcal infection in 1080 patients who received adalimumab therapy from 2003 to $2006 .{ }^{3)}$ The postmarketing database of the USFDA did not report any case of cryptococcal infection after adalimumab administration. ${ }^{10)}$ To date, only 2 cases suspected to be adalimumab-associated cryptococcosis have been reported in the English literature: all 2 cases involving the fingers. At present, our patient is the first case of opportunistic deep fungal infection involving the lung. However, adalimumab has not yet been used as widely as infliximab; therefore, it cannot be assumed that adalimumab is safer than infliximab or other TNF- $\alpha$ antagonists. The indication and the use of anti-TNF therapy with various new agents like adalimumab will spread rapidly and widely; therefore, such unexpected fungal infections could be encountered more frequently. Cryptococcus is an endemic infectious fungus that has often been isolated from the excreta of doves. Therefore, great care must be practiced by patients undergoing anti-TNF therapy while living or travelling in locations contaminated by such endemic fungi.

\section{Acknowledgements}

This study was funded by Kansai Rosai Hospital and Osaka City University Graduate School of Medicine. We thank Ms. Sae Hayashi for her help in preparing this manuscript.

\section{Disclosure Statement}

The authors have no conflicts of interest.

\section{References}

1) Tsiodras S, Samonis G, Boumpas DT, Kontoyiannis DP. Fungal infections complicating tumor necrosis factor alpha blockade therapy. Mayo Clin Proc 2008; 83: 181-94.

2) Wilson ML, Sewell LD, Mowad CM. Primary cutaneous cryptococcosis during therapy with methotrexate and adalimumab. J Drugs Dermatol 2008; 7: 53-4.

3) Horcajada JP, Pena JL, Martinez-Taboada VM, Pina T, Belaustegui I, et al. Invasive cryptococcosis and adalimumab treatment. Emerg Infect Dis 2007; 13: 953-5.

4) Winthrop KL. Risk and prevention of tuberculosis and other serious opportunistic infections associated with the inhibition of tumor necrosis factor. Nat Clin Pract Rheumatol 2006; 2: 602-10.

5) Herring AC, Lee J, McDonald RA, Toews GB, Huffnagle GB. Induction of interleukin-12 and gamma interferon requires tumor necrosis factor alpha for pro- 
tective t1-cell-mediated immunity to pulmonary cryptococcus neoformans infection. Infect Immun 2002; 70: 2959-64.

6) Huffnagle GB, Toews GB, Burdick MD, Boyd MB, McAllister KS, et al. Afferent phase production of tnfalpha is required for the development of protective $t$ cell immunity to cryptococcus neoformans. J Immunol 1996; 157: 4529-36.

7) U.S. Food and Drug Administration. FDA Requires Stronger Fungal Infection Warning for TNF Blockers. http://www.fda.gov/ForConsumers/ConsumerUpdates/ ucm107878.htm. Accessd: 29 Sep 2009.
8) Altz-Smith M, Kendall LG, Jr, Stamm AM. Cryptococcosis associated with low-dose methotrexate for arthritis. Am J Med 1987; 83: 179-81.

9) Law KF, Aranda CP, Smith RL, Berkowitz KA, Ittman MM, et al. Pulmonary cryptococcosis mimicking methotrexate pneumonitis. J Rheumatol 1993; 20: 872-3.

10) Schiff MH, Burmester GR, Kent JD, Pangan AL, Kupper H, et al. Safety analyses of adalimumab (humira) in global clinical trials and us postmarketing surveillance of patients with rheumatoid arthritis. Ann Rheum Dis 2006; 65: 889-94. 